\title{
Translational research is a key to nongeneticist physicians' genomics education
}

\author{
W. Gregory Feero, MD, PhD', Teri A. Manolio, MD, $\mathrm{PhD}^{2}$ and Muin J. Khoury, MD, PhD 3,4
}

Several recent publications detailing the clinical application of next-generation sequencing technologies mark a milestone in the journey from base pairs to the bedside. ${ }^{1,2}$ A technology assessment by a major insurer lends credence to the idea that the clinical use of sequencing technologies is no longer viewed as a research endeavor. ${ }^{3}$ The expansion of the clinical application of new sequencing technologies raises a wide range of complex issues in the health-care system. ${ }^{4}$ Recently, Manolio and Murray ${ }^{5}$ described the outcomes of a workshop convened by the National Human Genome Research Institute to discuss the growing opportunities for educating nongeneticist physicians and other health-care providers in genomics. As a result of the workshop, an Inter-Society Coordinating Committee on Practitioner Education in Genomics was formed to facilitate interactions among professional societies intended to increase the expertise of practitioners in applying genomics in clinical care. This represents a renewed US national attempt to increase genomics competency among a key group of critical decision makers. In this commentary, we explore some of the key contextual issues that are likely to mediate genomics educational demand and ultimately determine the success of genomics educational programs for nongeneticist health professionals, especially physicians.

Physicians comprise a highly heterogeneous population. In the United States alone, there are 24 medical specialties recognized by the American Board of Medical Specialties and hundreds of professional societies and organizations that play a role in the educational pipeline of physicians from undergraduate education through retirement. Widespread adoption of even simple interventions with the best evidence of health benefits-such as ensuring that aspirin and $\beta$-blockers are routinely employed in secondary prevention of coronary heart diseaseis surprisingly difficult to attain. Genomic science and the clinical technologies that have arisen from its application are dauntingly complex, leading a former director of the National Heart Lung and Blood Institute of the National Institutes of Health to quip regarding the translation of genomic discoveries to patient care, "If we didn't do it with aspirin, how can we expect to do it with DNA?"6 Genomics has the additional burden of attempting to rise to the surface in a tumult of healthcare system changes.

Recognition of the need to develop a health workforce competent in genetics is not new. In the 1970s, papers were published exploring the issue, and the topic has been cyclically revisited over the intervening decades, most notably around the inception and completion of the Human Genome Project. Interventions in education have taken a variety of forms, from calls to reform medical school curricula to professional society-sponsored continuing medical education efforts to US federal government funding of a health professional organization with the sole mission of furthering the genetic literacy of all health professionals. The majority of these efforts have had origins in the genetics and genomics community. Very likely, a dispassionate grading of the results of nearly 4 decades of efforts to improve the genetics literacy of nongeneticist physicians and other health professionals would yield low marks. Limited examples of genomics educational success exist, but numerous publications document that the majority of physicians remain interested in genomics but lacking confidence and facility with the topic.

We feel that the fundamentals of the equation of cost versus benefit of attaining genomics competency for many physicians are shifting in favor of genomics education. This is most evident in the area of oncology, where the availability of new targeted therapies paired with knowledge of the genomic drivers of an individual patient's tumor are improving outcomes for a wide variety of common cancers. This has translated to the extensive genomic education content offered at the 2014 meeting of the American Society of Clinical Oncology. The increasing alignment of incentives for provision of proven preventive services favors development of genomic approaches to risk stratification, as evidenced by the inclusion of genomics as a topic in the US Healthy People 2020 objectives. The Office of Public Health Genomics has introduced a three-tier classification system and continually updated listing of genomics applications. Applications falling into tier 1 have evidence-based guidelines or substantial evidence

\footnotetext{
${ }^{1}$ Maine Dartmouth Family Medicine Residency Program, Augusta, Maine, USA; ${ }^{2}$ Division of Genomic Medicine, National Human Genome Research Institute, National Institutes of Health, Bethesda, Maryland, USA; ${ }^{3}$ Office of Public Health Genomics, Centers for Disease Control and Prevention, Atlanta, Georgia, USA; ${ }^{4}$ Epidemiology and Genomics Research Program, Division of Cancer Control and Population Sciences, National Cancer Institute, National Institutes of Health, Rockville, Maryland, USA. Correspondence: W. Gregory Feero (wfeero@mainegeneral.org)
} 
supporting their use; tier 2 applications have sufficient evidence supporting clinical utility to consider use; tier 3 includes technologies for which there is little, if any, evidence supporting benefit or existing guidelines recommending against use. Tier 1 is, at this time, dominated by cancer-related genomic applications. ${ }^{7}$ It seems quite likely that consumer information demand for healthrelated technologies will drive increased information seeking on the part of physicians. Physicians and other health professionals already have much catching up to do to improve their understanding of genomics.

Available studies suggest that development and maintenance of freely available, high-quality genomics reference and educational materials is likely insufficient to ensure a meaningful increase in genomics competency among nongeneticist health providers. ${ }^{8,9}$ Meaningful increases in genomics competency will require both cultural and infrastructure changes that nurture and sustain a demand for knowledge regarding genomic advances capable of improving patient-oriented outcomes. The success of any major genomics educational effort for health professionals will hinge on the ability to effectively negotiate three realities that confront any new health-care technology attempting to gain wide adoption in the United States: lack of evidence demonstrating value, incompatibility across health information technology/electronic health record systems, and the ever-growing time burden facing health professionals in the clinic (Figure 1).

First, current health-care reform efforts have improved outcomes at a reasonable cost (value) as a core principle. Healthcare professionals are increasingly expected and incentivized to choose high-value interventions; those with low or unclear value are unlikely to compete well for clinical use, an obvious driver

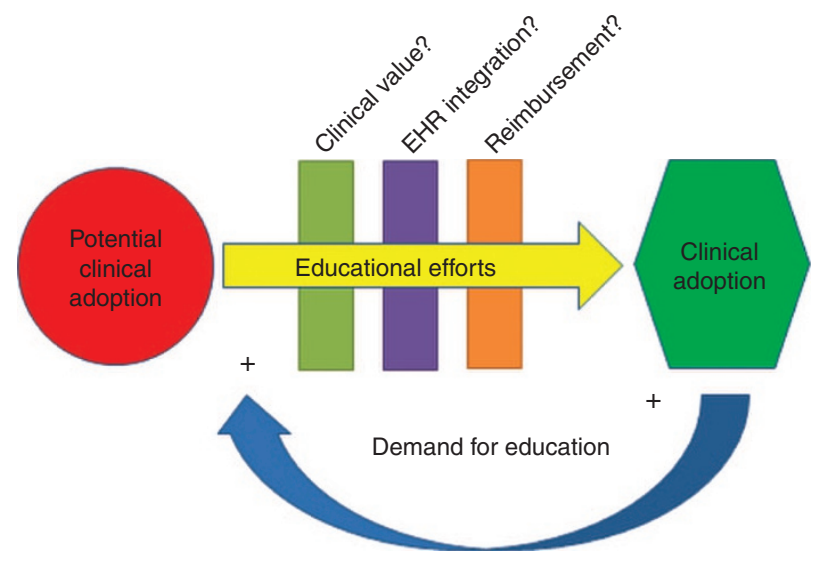

Figure 1 Adoption of genomic technology in clinical care requires education of health-care professionals. Education efforts (yellow arrow) face several contextual challenges that reduce potential health professional interest. First is the need to ensure that new technologies have adequate evidence supporting their value in improving health outcomes (green rectangle). Second, genomic technology must produce information that is compatible with existing electronic health record systems (purple rectangle). Third, health teams will need to be reimbursed adequately for the time required to integrate genomic information into patient care (orange rectangle). Increased clinical use of genomic technology is likely to have a positive effect on educational demand (blue arrow) and educational efforts as the number of providers exposed to genomics in clinical care increases. for educational demand. Translational research in genomics remains largely in the realm of early preclinical studies, and a lack of clinical studies that conclusively demonstrate value precludes inclusion of promising genomic technologies in quality metric development and incentive programs. There is a growing backlog of potentially beneficial genomic applications with little available data regarding real-world effectiveness or comparative effectiveness (tiers 2 and 3 in the Centers for Disease Control and Prevention's classification scheme). Alignment of payment incentives to favor applications with demonstrated clinical utility would increase the probability that applications come to market, especially if comparative effectiveness suggests a competitive advantage over older, lower-value technologies. Establishment of the Patient-Centered Outcomes Research Institute may also help to address this backlog.

Second, electronic health records remain largely ineffectual for the management and interpretation of genomic information, including family health history data. Standards for some types of genomic data have been developed and adopted by major standards development organizations such as Health Level 7. However, commercial platforms have been slow to incorporate the standards or to facilitate the flow of structured information across systems necessary for automated genomic data interpretation and clinical decision support. Absent access to effective electronic support for the storage, exchange, interpretation, and use of genomic data, interest in educational efforts will be severely limited by the impracticality of using electronic systems outside of electronic health records to manage genomic data. The Meaningful Use process of the US Centers for Medicare and Medicaid Services is intended to help drive innovation in electronic health records and adoption of improved electronic functionalities, but to date little emphasis has been placed on developing the facility to handle genomics data.

Third is the need for adequate reimbursement for the time required to incorporate genomic services into patient care. Clinicians and health-care teams cannot deliver an infinite number of time-intensive services to patients without adequate reimbursement. Conceivably, comparative effectiveness research results might suggest replacement of some current practices with newer genomic technologies. However, genomics poses unique knowledge and communication issues for care providers and their patients; even with a high degree of genomics literacy and some degree of improved automation, genomic information will add to the time pressures a care team faces. As delivery structures for care such as the patient-centered medical home and payment models based around accountable care organizations are developed, this real cost in time to the care team must be accounted for. Improved use of information technology systems for patient and provider education might reduce some of the time burden in the office setting, but such sophisticated infrastructure is expensive. The catch-22 of this situation is that in many environments, key decision makers developing new models of care likely lack sufficient genomics education to plan for the potential advances genomics might bring to health care over the next 2 decades. Absent reimbursement for the time required to effectively 
communicate with patients regarding genomic interventions, physicians will likely choose to deliver (and learn about) less time-consuming interventions with demonstrated value.

Advancements in genomic science, coupled with changes in the health-care landscape, have opened potential opportunities for improved patient care and disease prevention that can only be fully capitalized on by an educated health professional, and in particular, physician, workforce. Developing such a workforce will require substantial, focused, and sustained resource commitment from both the public and the private sectors. ${ }^{10}$ Perseverance and cooperation across the entire health-care and biomedical research ecosystem are needed to ensure that educational efforts succeed and that the full measure of health benefits from genomic science are achieved.

\section{DISCLOSURE}

The authors declare no conflict of interest. The corresponding author created this work as part of his official responsibilities as an Intergovernmental Personnel Act appointee with the National Cancer Institute, National Institutes of Health.

\section{REFERENCES}

1. de Ligt J, Willemsen MH, van Bon BW, et al. Diagnostic exome sequencing in persons with severe intellectual disability. N Engl J Med 2012;367: 1921-1929.

2. Yang Y, Muzny DM, Reid JG, et al. Clinical whole-exome sequencing for the diagnosis of mendelian disorders. N Engl J Med 2013;369: 1502-1511.

3. Special Report: Exome Sequencing for Clinical Diagnosis of Patients with Suspected Genetic Disorders. http://www.bcbs.com/blueresources/tec/ vols/28/28_03.pdf. Accessed 19 December 2013.

4. Dewey F. Clinical interpretation and implications of whole-genome sequencing. JAMA 2014;311:1035-1045.

5. Manolio TA, Murray MF. The growing role of professional societies in educating clinicians in genomics. Genet Med 2014;16:571-572.

6. Lenfant C. Shattuck lecture-clinical research to clinical practice-lost in translation? N Eng/ J Med 2003;349:868-874.

7. Classification of Genomic Applications by Levels of Evidence. http://www.cdc. gov/genomics/gtesting/tier.htm. Accessed 19 December 2013.

8. Carroll JC, Wilson BJ, Allanson J, et al. GenetiKit: a randomized controlled trial to enhance delivery of genetics services by family physicians. Fam Pract 2011;28:615-623.

9. Martin G, Currie G, Finn R. Bringing genetics into primary care: findings from a national evaluation of pilots in England. J Health Serv Res Policy 2009;14: 204-211.

10. Frieden TR. Six components necessary for effective public health program implementation. Am J Public Health 2014;104:17-22. 